\title{
CONTROL LAW DESIGN IN A COMPUTATIONAL AEROELASTICITY ENVIRONMENT
}

Jerry R. Newsom

NASA LaRC

Hampton, VA

757-864-6051

\author{
Harry H. Robertshaw \\ Virginia Tech \\ Blacksburg, VA \\ 540-231-7196
}

\author{
Rakesh K. Kapania \\ Virginia Tech \\ Blacksburg, VA \\ 540-231-4881
}

\begin{abstract}
A methodology for designing active control laws in a computational aeroelasticity environment is given. The methodology involves employing a systems identification technique to develop an explicit statespace model for control law design from the output of a computational aeroelasticity code. The particular computational aeroelasticity code employed in this paper solves the transonic small disturbance aerodynamic equation using a time-accurate, finitedifference scheme. Linear structural dynamics equations are integrated simultaneously with the computational fluid dynamics equations to determine the time responses of the structure. These structural responses are employed as the input to a modern systems identification technique that determines the Markov parameters of an "equivalent linear system". The Eigensystem Realization Algorithm is then employed to develop an explicit state-space model of the equivalent linear system. The Linear Quadratic Guassian control law design technique is employed to design a control law. The computational aeroelasticity code is modified to accept control laws and perform closed-loop simulations. Flutter control of a rectangular wing model is chosen to demonstrate the methodology. Various cases are used to illustrate the usefulness of the methodology as the nonlinearity of the aeroelastic system is increased through increased angle-of-attack changes.
\end{abstract}

\section{INTRODUCTION}

Aeroelasticity is the mutual interaction between aerodynamics and a flexible body. Aeroelasticity has been and continues to be an extremely important consideration in many aircraft designs ${ }^{1}$. To address undesirable aeroelastic effects or phenomena, the stiffness of the wing is often increased, adding weight to the aircraft and decreasing the overall performance.

The control of aeroelastic response through feedback to control surfaces, or more recently through feedback to active materials, is an alternative to "passive control" through increased stiffness. $\mathrm{Noll}^{2}$ presents a review of active control methods, wind-tunnel experiments, and flight experiences associated with feedback control and aeroelasticity. Currently, most aeroelastic analyses are routinely conducted using linear aerodynamic and linear structural models. However, within the last few decades, a significant increase in advancing methods to consider nonlinear aeroelasticity, especially nonlinear aerodynamics in the transonic region, has taken place. Dowell $^{3}$ presents an excellent overview of nonlinear aeroelasticity and major problems where nonlinear effects should be considered.

With the maturity of CFD codes, their incorporation into aeroelastic analyses, both static and dynamic, is beginning to occur, primarily in the research community. This relatively new field has been termed "computational aeroelasticity" and involves coupling structural elasticity (static and/or dynamic) and computational fluid dynamics together to perform time domain analyses, where the aerodynamics are a nonlinear function of the deformation of the aircraft. Most of the work in computational aeroelasticity has employed solutions to either the nonlinear potential equation or the Euler equation for aerodynamic forces. Bennett and Edwards ${ }^{4}$ provide a status of recent developments in computational aeroelasticity, with an emphasis on unsteady transonic flow, and results of some applications.

Computational aeroservoelasticity involves coupling structural dynamics, computational fluid dynamics, and active control systems together. Batina and Yang were perhaps the first researchers to examine control of an aeroelastic system in a computational aeroelasticity environment for transonic flow. They conducted studies with a 2-d airfoil with plunge and pitch degrees-of-freedom and a 2-d small-disturbance transonic CFD code. The effect of a simple constant gain control law utilizing displacement, velocity, and acceleration feedback on the time responses was determined. Comparison with linear theory indicated that the frequency and damping values were significantly different for transonic and linear subsonic 
theory results. References 6-10 are other examples of research in control of aeroelastic systems within a computational aeroelasticity environment. Similar to Batina and Yang, these studies also only involve varying the gains of simple feedback control laws to study their effect on the response of the aeroelastic system. Two-dimensional and three-dimensional small-disturbance and Euler CFD codes are used in these studies. The studies show that feedback control can be effective in suppressing transonic flutter.

Guillot and Friedman ${ }^{11,12}$ employed adaptive control theory to design control laws using a CFD technique. A 2-d airfoil model, with a trailing-edge control surface, was used with an Euler CFD code to perform the computational aeroelastic solutions. An adaptive control law was used because of the assumed nonlinear behavior of the system in the presence of nonlinear transonic flow with large shock motions. The adaptive control law involved identifying a linear autoregressive moving average (ARMA) model and then determining an optimal full-state control law. At the end of a learning period, the control law was engaged and the ARMA model and control law were then updated at each subsequent time step during the computational aeroelasticity simulation. An adaptive control law was shown to be quite effective in suppressing transonic flutter with strong shocks.

The objective of this paper is to describe a general methodology to design control laws in the context of a computational aeroelasticity environment. The technical approach involves employing a systems identification technique to develop an explicit statespace model for control law design from the output of a computational aeroelasticity code. Although there are many control law design techniques available, the standard Linear Quadratic Guassian technique is employed in this paper. The computational aeroelasticity code is modified to accept control laws and perform closed-loop simulations. Numerical results for flutter suppression of the Benchmark Active Control Technology wind-tunnel model are given to illustrate the approach.

\section{DESCRIPTION OF TECHNICAL APPROACH}

The overall methodology begins with performing a computational aeroelasticity simulation (uncontrolled) with prescribed control surface inputs to obtain a set of corresponding output time histories. The next step is to employ a system identification technique, using the time histories of outputs and inputs from the first step, to determine an "equivalent linear system" for use as a control law design model. Next, design of a control law can be performed using any control law design technique. Finally, the control law is evaluated in the computational aeroelasticity simulation. If the control law performance is not adequate, the control law can be redesigned and evaluated again until the desired performance is obtained. A detailed description of the technical approach is given in Ref. 13. A brief overview will be given in this paper.

\section{Computational Aeroelasticity Simulation}

Computational aeroelasticity simulation involves integrating the structural, aerodynamic (CFD), and for this paper, control equations simultaneously. This paper focuses on the transonic case where the aerodynamic fluid flow equations contain nonlinear terms. The particular code that is employed is the CAP-TSD ${ }^{14}$ code that has been developed at the NASA Langley Research Center. The CAP-TSD code is a finite-difference code which solves the transonic smalldisturbance aerodynamic equation. The primary outputs of the CAP-TSD code are time histories of the pressures and the generalized coordinate displacements, velocities, and accelerations. It has been used on a wide variety of configurations for steady and unsteady pressure distribution calculations and for calculating transonic flutter characteristics, including nonlinear limit-cycle instabilities.

The basic equations of motion implemented in CAPTSD are;

$$
[M]\{\ddot{q}\}+[C]\{\dot{q}\}+[K]\{q\}=\{F\}
$$

The primary difference between linear aeroelasticity methods and computational aeroelasticity is in the computation of the aerodynamic pressure coefficient $C_{p}$ used in computing the generalized aerodynamic force vector $\{F\}$.

The CAP-TSD code is a finite difference code that solves the general-frequency modified TSD potential equation. The TSD equation is solved within CAPTSD by a time-accurate approximate factorization (AF) algorithm developed by Batina ${ }^{15}$. The algorithm consists of a Newton linearization procedure coupled with an internal iteration technique. The CAP-TSD code is capable of treating configurations with 
multiple lifting surfaces and bodies. A relatively simple Cartesian grid is input along with the coordinates defining the geometry of the configuration and the corresponding surface slopes. The pressure coefficient is calculated at each time step and is employed to calculate the generalized force vector at each time step.

Equation 1 is rewritten in state-space form as

$$
\{\dot{X}\}=[A]\{X\}+[B]\{u\}
$$

The numerical algorithm, employed in CAP-TSD, for solving equation 2 is

$$
X_{n+1}=\phi X_{n}+\theta B\left(3 u_{n}-u_{n-1}\right) / 2
$$

where

$$
\begin{aligned}
& \phi=e^{A \Delta T} \\
& \theta=\int_{0}^{\Delta T} e^{A(\Delta T-\tau)} d \tau
\end{aligned}
$$

and $\Delta \mathrm{T}$ is the integration time step.

\section{Control Design Model Development}

Most control law design methods require an explicit mathematical model of the system to be controlled. A computational aeroelasticity simulation provides time histories of the variables of an aeroelastic system, but does not generate an explicit mathematical model of the system. Computational aeroelasticity simulations are analogous to performing experimental investigations where the only direct outputs are time responses. Therefore, to employ the various control law design methods that are available, a control design mathematical model of a "computational aeroelasticity system" (CAS) must be developed. System identification techniques are widely employed for developing a mathematical model given experimental data. Therefore, since a CAS simulation is analogous to performing an experiment, system identification is a logical choice. Juang $^{16}$ provides a description of many of the advances in system identification for vibration modal testing and control of space structures. The Observer/Kalman filter Identification (OKID) technique ${ }^{17}$ was developed primarily for development of a mathematical model for control law design. The
OKID technique has been applied to space structures, such as the Hubble Space Telescope ${ }^{16}$ and the Shuttle Remote Manipulator System ${ }^{18}$, and to the identification of linear rigid aircraft models ${ }^{19}$. Because the OKID technique was developed primarily for identifying models for control law design, it is the technique employed in this paper.

Description of Observer/Kalman Filter Identification Technique. Given a set of input and output data, the Observer/Kalman Filter Identification (OKID) algorithm is shown in Fig. 1. One of the keys to the OKID algorithm is the introduction of an observer into the identification process. The fist step of the process is the calculation of the observer Markov parameters. Then the system Markov parameters are obtained.

Consider a discrete time state-space model of a system described by a set of first order difference equations of the form

$$
\begin{aligned}
& x(k+1)=\hat{A} x(k)+\hat{B} u(k) \\
& y(k)=C x(k)+D u(k)
\end{aligned}
$$

Solving for the output $y(k)$ in terms of the previous inputs, with the assumption that the system is initially at rest, i.e. $x(0)=0$, yields

$$
y(k)=\sum_{i=0}^{k} h_{i} u(k-i)
$$

where the parameters

$$
h_{0}=D, \quad h_{k}=C \hat{A}^{k-1} \hat{B}, \quad k=1,2,3, \ldots
$$

are the system Markov parameters, which are also the system pulse response samples. To reduce the number of Markov parameters needed to adequately model a system, an observer is introduced into the OKID technique. Adding and subtracting the term $\bar{K} y(k)$ to the right hand side of equation (4) yields

$$
\begin{aligned}
& x(k+1)=(\hat{A}+\bar{K} C) x(k)+ \\
& (\hat{B}+\bar{K} D) u(k)-\bar{K} y(k) \\
& y(k)=C x(k)+D u(k)
\end{aligned}
$$

The matrix $\bar{K}$ can be interpreted as an observer gain. The parameters defined as 


$$
\begin{aligned}
\bar{Y}(k) & =C(\hat{A}+\bar{K} C)^{k-1}[\hat{B}+\bar{K} D,-\bar{K}] \\
& =\left[\beta_{k}, \quad \alpha_{k}\right]
\end{aligned}
$$

are the Markov parameters of an observer system. Consider the special case where $\bar{K}$ is a deadbeat observer gain such that all eigenvalues of $\hat{A}+\bar{K} C$ are zero, the observer Markov parameters will become identically zero after a finite number of terms. For lightly damped systems, this means that the system can be described by a reduced number of observer Markov parameters. Furthermore, an unstable system can be represented using this technique. This is obviously a major advantage for this research.

After the Markov parameters are computed using a least squares technique, a state-space model of the system is developed using the Eigensystem Realization Algorithm (ERA). A complete description for the computation of the Markov parameters and the ERA is given in Refs. 17 and 20.

Control Design. There are many control law design methods available. These range from classical control law design to the LQG method to $H_{\infty}$ robust control law design methods to nonlinear control law design methods. Because of its ease of use, the LQG design method is employed in this paper. Basically, the LQG design process involves minimizing a cost function of the form

$$
J=\int_{0}^{\infty}\left[y^{T} Q y+u^{T} R u\right] d t
$$

where $y$ is an output vector (e.g. accelerations, loads) of the system and $u$ is the control input. The resulting control law is $\mathrm{u}=-\mathrm{Gx}$ where $\mathrm{G}$ is the state feedback gain matrix that minimizes the cost function and $\mathrm{x}$ is the state vector. Since the states of a system are generally not all available for feedback, a Kalman filter is employed to estimate the states. The resulting control law is of the form:

$$
\begin{aligned}
& \{\hat{\dot{x}}\}=[\bar{A}]\{\hat{x}\}+[L]\{y\} \\
& \{u\}=[-G]\{\hat{x}\}
\end{aligned}
$$

where $\{\hat{x}\} \quad \begin{aligned} & \text { is the estimate of the state vector } \mathrm{x} \\ & \text { is the Kalman filter gain matrix. }\end{aligned}$
and $\mathrm{L}$

\section{RESULTS AND DISCUSSION}

The example employed in this paper to demonstrate the overall control law design methodology is active flutter suppression for the Benchmark Active Controls Technology (BACT) ${ }^{21}$ wind-tunnel model shown in Fig. 2. The BACT model is a rigid, rectangular wing with a NACA 0012 airfoil section. It is equipped with a trailing-edge control surface and upper and lower surface spoilers that are controlled independently by hydraulic actuators. Only the trailing-edge control surface is employed in this paper. For this study, actuator dynamics are ignored. For the control law designs, an accelerometer located near the outboard trailing edge is the assumed sensor employed for feedback. The wing is mounted to a device called the Pitch and Plunge Apparatus (PAPA) which is designed to permit motion in principally two modes - pitching and vertical translation (plunge). Therefore, the BACT has structural dynamic behavior very similar to the classical two degree-of-freedom problem in aeroelasticity. The vibration frequencies, computed from a NASTRAN model of the BACT, are $3.4 \mathrm{~Hz}$ (plunge) and $5.2 \mathrm{~Hz}$ (pitch). Structural damping is assumed to be zero. There are two CAP-TSD aerodynamic representations of the BACT wind-tunnel model used in this paper. The first one, shown in Fig. 3, is a 3-d model where the CFD grid contains 140 points in the chordwise direction, 40 points in the spanwise direction, and 92 points in the vertical direction for a total of 515,200 grid points. The second one is an equivalent 2-d model where the CFD grid contains 140 points in the chordwise direction, only 2 points in the spanwise direction, and 92 points in the vertical direction for a total of 25,760 grid points.

Although some basic aerodynamic and flutter calculations will be presented for the 3-d model, the control law design and evaluation process results will be demonstrated with the 2-d model. However, the exact same process would be applied to the 3-d case.

\section{Basic Aerodynamic and Uncontrolled Flutter Results}

In order to calculate rigid aerodynamic pressures, the fluid flow equation (TSD potential equation) is integrated in time by CAP-TSD without coupling the structural dynamics equations (i.e. no aeroelastic effects). Figure 4 shows the chordwise pressure 
distribution at the $60 \%$ span location at $M=0.77$ for 2 degrees angles of attack. Results from CAP-TSD are shown with the experimental results of Ref. 21. Linear CAP-TSD results are also shown. At 2 degrees angle of attack, a weak shock near the $20 \%$ chord has developed indicative of transonic flow. At 5 degrees angle of attack (not shown), the shock moves aft to approximately the $35 \%$ chord and becomes moderately strong. The CAP-TSD results capture the shock location and strength reasonably well. Of course, the linear CAP-TSD results do not indicate a shock and are not in agreement with the experimental data on the upper surface forward of the shock. However, both the CAP-TSD and linear CAP-TSD results compare very well with the experimental data aft of the $40 \%$ chord on the upper surface and compare well along the entire chord for the lower surface. The apparent dip in the experimental upper surface pressures near the leading edge has been attributed to the transition strip at that location ${ }^{21}$. These results provide some degree of confidence in the aerodynamic predictions of CAP-TSD for angle-of-attack changes.

Figure 5 shows the chordwise pressure distribution at the $60 \%$ span location at $M=.77$ for trailing-edge control surface deflections of 2 degrees. Again, results from CAP-TSD are shown with the experimental results of Ref. 21. CAP-TSD slightly overpredicts the supper surface pressure peak near the control surface hinge line. CAP-TSD considerably (approximately 50\%) overpredicts the upper surface pressure peak for $\delta=5$ degrees (not shown). Overprediction of control surface forces is typical for inviscid codes. Therefore, care should be used in real applications to account for this overprediction when using an inviscid code.

For dynamic aeroelastic analyses in a computational aeroelasticty environment, two steps are employed in performing the calculations ${ }^{22}$. In the first step, a static aeroelastic deformation is calculated to provide the initial flowfield for the dynamic aeroelastic solution. The dynamic solution is a perturbation about a converged static aeroelastic solution for each Mach number and dynamic pressure of interest. The procedure for calculating static aeroelastic solutions is to allow the structure and aerodynamics to interact with no initial condition on the modal displacements and velocities and no external excitation. A very large value (0.99) for structural damping is used to prevent divergence of the solution and to accelerate convergence. Once a static aeroelastic solution is computed, the next step is to prescribe either an initial condition on the modal displacements or velocities or an external input. For flutter calculations, initial conditions on the velocities are used to begin the dynamic structural integration. CAP-TSD was run using a value of 1 on both the dimesionless plunge and pitch modal velocity initial conditions and then allowed to decay freely. The ERA system ID method of Ref. 20 was employed to estimate modal dampings and frequencies at various values of dynamic pressure. Figure 6 shows the dampings as a function of dynamic pressure $(M=0.77)$ for a Doublet Lattice linear model, the CAP-TSD 3-d linear model, and CAP-TSD 3-d model.

The CAP-TSD 3-d linear model shows approximately a $9 \%$ greater flutter dynamic pressure than the Doublet Lattice linear model. The Doublet Lattice linear model shows approximately a 3\% lower flutter dynamic pressure than the experimental value. The CAP-TSD linear model shows approximately a $6 \%$ greater flutter dynamic pressure than the experimental value. The CAP-TSD model shows approximately a $2 \%$ greater flutter dynamic pressure than the experimental value.

\section{CAP-TSD 2-d Results}

This section presents uncontrolled flutter results, initial system identification results, and results illustrating the sensitivity of the identified system model. All of the results are for a Mach number of 0.77 and employ the CAP-TSD 2-d model previously described. All of the CAP-TSD simulation results were calculated with an integration time increment of .000173 seconds and 8000 time steps.

Uncontrolled Flutter Results The ERA system ID method was also employed to estimate modal dampings and frequencies for the 2-d cases. The overall character of the damping vs. dynamic pressure curves (fig. 7) is very similar to the 3 -d case. The CAP-TSD linear 2-d model shows a flutter dynamic pressure which is approximately $19 \%$ lower than the corresponding linear 3-d case. The CAP-TSD 2-d model shows a flutter dynamic pressure that is approximately $21 \%$ lower than the corresponding nonlinear 3-d case. This magnitude of reduction between 3-d and 2-d is similar to that described in Ref. 23.

Initial System ID Results In order to perform a system identification (ID), an appropriate control surface input signal must be used to excite the system 
modes. After exploring several input signals (sinusoid sweep and random), an exponential pulse signal provided a good input signal for identifying an equivalent linear model of the CAP-TSD outputs. In order to test the system ID technique, CAP-TSD was run in the linear mode for several of the initial results that will be shown. A dynamic pressure of $5.75 \mathrm{kPa}$ was chosen to identify all of the 2-d system ID models. This dynamic pressure is between the flutter dynamic pressure predicted by CAP-TSD (linear) and the flutter dynamic pressure predicted by CAP-TSD (nonlinear). The linear system ID models were identified using the plunge and pitch displacements, velocities, and accelerations as the measurement signals. Figure 8 shows the results from the system ID procedure in terms of a comparison between the CAP-TSD outputs (linear) and two system ID model outputs (using the same exponential pulse input).

A 5-state system ID model was the first one identified. This 5-state model matches the CAP-TSD plunge responses well. However, it does not match the CAP-TSD pitch response well during the exponential pulse time period. The order of the system ID model was increased and an 8-state model was identified. As can be seen in Fig. 8, the 8-state system ID model provides a very good representation of the CAP-TSD results for both the plunge and pitch responses over the entire time history. Several additional system ID models with higher orders were identified, but no overall improvement (over the 8state model) in the ability to match the CAP-TSD results were seen. Most of the subsequent results employ an 8-state system ID model.

In order to assess the sensitivity of the system ID model to changes in the control input signal, the system ID model (derived from the exponential pulse input) was used in a simulation where the control surface input was a sine wave. This result employed the outputs of CAP-TSD run in the nonlinear mode (including the aerodynamic nonlinear terms). Both the outputs used as input to system ID procedure and the simulation for the sine wave input were for the nonlinear CAP-TSD mode. Figure 9 shows results of the simulation using a $5 \mathrm{~Hz}$ and $10 \mathrm{~Hz}$ sine wave input in terms of a comparison of the system ID model with the CAP-TSD output. Only the plunge and pitch displacements are shown. The agreement between the system ID model and the CAP-TSD outputs are excellent for the $5 \mathrm{~Hz}$ case. There is some slight mismatch of the peaks (especially for the pitch displacement) between the system ID model and the CAP-TSD outputs for the $10 \mathrm{~Hz}$ case. However, the overall ability of the system ID model to match the CAP-TSD outputs for these different inputs is quite good.

\section{Control Law Design and Evaluations}

Five different cases will be presented to illustrate the design methodology. All of the cases are for a Mach number of 0.77 and a dynamic pressure of $5.75 \mathrm{kPa}$. The cases begin with the linear case at 0 degrees angle of attack and then proceed with nonlinear cases at 0 degrees angle of attack and 0.6 degrees angle of attack. For each angle of attack, a system-ID model is developed and the control law designed with the system ID model for that angle of attack is compared with the control law for the linear case. The comparison is in terms of gain and phase margins, acceleration time history (using the exponential control input), and control surface deflection. The control law designed with the linear model is intended to represent a control law using the state-of-the-art methodology.

The primary design goal for all of the control law designs is to increase the damping of the system while exhibiting at least $6 \mathrm{~dB}$ gain margins and 60 degrees phase margins. The gain and phase margins are to account for uncertainty in the model. In addition, the control surface displacements, due to the feedback command, should be less than 1 degree in order to stay within a somewhat linear range of the control surface displacement. In each of the LQG designs, the weighting on the output and input during the regulator design and the intensities of the noise matrices during the Kalman filter design were varied by trial and error until a design that met the goal was determined.

Case 1 Case 1 involves developing a system ID model from the CAP-TSD (in a linear mode) outputs, designing an LQG control law, and evaluating the LQG control law using CAP-TSD in the linear mode. The results of the system ID process were previously described.

Figure 10 shows a comparison of CAP-TSD linear and the system ID linear model outputs for the uncontrolled and controlled (using the LQG control law) case. The results using the system ID model and the CAP-TSD simulation for the controlled case are almost identical. The controlled case indicates a much higher degree of damping than the uncontrolled case. 
A gain margin of $8.52 \mathrm{~dB}$ and a phase margin of 76.28 degrees were determined using a Bode plot (not shown) of the open-loop system (system ID model and LQG control law). Figure 10 also shows the feedback control surface command for Case 1. The maximum control surface displacement is approximately 0.3 degrees and occurs during the exponential pulse excitation.

Case 2. Case 2 involves evaluating the control law designed in Case 1 using the CAP-TSD nonlinear simulation at an angle of attack of 0 degrees. In order to calculate stability margins and design a control law using the results of the CAP-TSD nonlinear simulation (Case 3), the CAP-TSD (nonlinear) outputs are used as the input to the system ID procedure. Figure 11 shows the results from the system ID procedure in terms of a comparison between the CAP-TSD (nonlinear) displacement outputs and the system ID model. Similar to the linear case, the system ID model provides a very good representation of the CAP-TSD outputs.

Figure 12 shows a comparison of CAP-TSD outputs for the uncontrolled and controlled (using Case 1 control law) case. The controlled response using the CAP-TSD nonlinear model is not as highly damped as the linear model results. A gain margin of -4.75 $\mathrm{dB}$ and a phase margin of 31.34 degrees were determined using a Bode plot (not shown) of the open-loop system Although stable, the control law designed using the linear model does not provide satisfactory stability margins. Figure 12 also shows the feedback control surface command for Case 2 . Similar to the Case 1, the maximum control surface displacement is approximately 0.3 degrees and occurs during the exponential pulse excitation.

Case 3 Case 3 employs the system ID model derived from the nonlinear CAP-TSD results of Case 2 to design a control law. After some trial and error during the LGQ design process, a satisfactory control law was determined. Figure 13 shows a comparison of CAP-TSD outputs for the uncontrolled and controlled case. A gain margin of $-10.84 \mathrm{~dB}$ and a phase margin of 64.72 degrees were determined using a Bode plot (not shown) of the open-loop system. Figure 13 also shows the feedback control surface command for Case 3. Similar to the Cases 1 and 2, the maximum control surface displacement is approximately 0.3 degrees and occurs during the exponential pulse excitation. The results using this control law clearly show much better results, in particular stability margins, than using the control law designed using the system ID model of the linear CAP-TSD outputs.

Case 4 Case 4 involves evaluating the control law designed in Case 1 using the CAP-TSD nonlinear simulation at an angle of attack of 0.6 degrees. In order to evaluate the stability margins and design a control law for Case 5, the system ID procedure is applied to the outputs of CAP-TSD at 0.6 degrees angle of attack. Obtaining a good system ID model for this case was a significant challenge because of the sensitivity of the system ID procedure to a perceived bias (nonzero value at $t=0$ ), sensitivity to the order of the model, and sensitivity to the value of the sampling time. In order to obtain a good system ID model, it was necessary to increase the sampling time by a factor of forty (decrease the sample rate by a factor of forty). When the perceived bias was removed, the sample rate was decreased and the model order was 5, a good system ID model was obtained. Figure 14 shows the results from the system ID in terms of a comparison between the CAP-TSD outputs and the system ID model. Although the system ID model provides a good representation of the CAP-TSD results, it is not as good as the 0 degree angle of attack case.

Figure 15 shows a comparison of CAP-TSD outputs for the uncontrolled and controlled (using Case 1 control law) case. The controlled system can be seen to be slightly unstable. Figure 15 also shows the feedback control surface command for Case 4 . The unstable character is clearly seen in the feedback control surface command.

Case 5 Case 5 employs the system ID model derived from the nonlinear CAP-TSD outputs at 0.6 degrees angle of attack to design a control law. Figure 16 shows a comparison of CAP-TSD outputs for the uncontrolled and controlled case. There is a significant reduction in the acceleration response with the controlled case. A gain margin of $-9.66 \mathrm{~dB}$ and a phase margin of 64.17 degrees were determined using a Bode plot (not shown) of the open-loop system. Figure 16 also shows the feedback control surface command for Case 5. Similar to the previous cases, the maximum control surface displacement is approximately 0.3 degrees and occurs during the exponential pulse excitation. The results using this control law clearly show much better results, in 
particular stability margins and damping, than using the control law designed using the system ID model of the linear CAP-TSD outputs.

\section{CONCLUSIONS}

In summary, the objective of this paper was the development of a general methodology for designing active control laws in a computational aeroelasticity environment. The methodology involves using a modern system identification technique to develop an equivalent linear model from the nonlinear simulation results. Standard control law design techniques are then used to design control laws. The resulting control laws are then incorporated into the nonlinear computational aeroelasticity simulation for evaluation. Results of a numerical study applying this methodology to flutter control of the BACT wind-tunnel model were presented.

The major conclusions of this research are:

1. Equivalent linear models developed by employing a system identification technique can represent the input-output relationship of a computational aeroelasticity simulation very well.

2. For the BACT model used in this study, the system ID model represents the input-output relationship very well until an angle of attack of .6 degrees where the transonic flow conditions cause the shock on the upper surface to move aft of the $40 \%$ chord. At this point, extreme care was needed to obtain a good system ID model.

3. A control law designed using a system ID model developed from a nonlinear simulation can control the nonlinear model better than a control designed using a system ID model developed from a linear computational aeroelasticity simulation.

\section{$\underline{\text { References }}$}

[1] Friedman, P. P.: "The Renaissance of Aeroelasticity and Its Future," SDM Lecture, 38th AIAA/ASME/ASCE/AHS/ASC Structures, Structural Dynamics and Materials Conference, April 1997.

[2] Noll, T. E.: "Flight-Vehicle Materials, Structures, and Dynamics - Assessment and Future Directions," Volume 5, 1993, pp. 179 - 212.

[3] Dowell, E. H.: "Nonlinear Aeroelasticity," AIAA-90-1031-CP, April 1990.

[4] Bennett, R. M. and Edwards, J. W.: "An Overview of Recent Developments in Computational Aeroelasticity," AIAA 98-2421, June 1998.

[5] Batina, J. T. and Yang, T. Y.: "Transonic Calculation of Airfoil Stability and Response With Active Controls," AIAA 84-0873, April 1984.

[6] Guruswamy, G. P., Tu, E. L., and Goorjian, P. M.: "Transonic Aeroelasticity of Wings with Active Control Surfaces," AIAA 87-0709-CP, April 1987.

[7] Ominsky, D. and Ide, H.: "An Effective Flutter Control Method Using Fast, Time-Accurate CFD Codes," AIAA 89-3468-CP, April 1989.

[8] Bendiksen, O., Hwang, G., and Piersol, J.: "Nonlinear Aeroelastic and Aeroservoelastic Calculations for Transonic Wings," AIAA 98-1898, April 1998.

[9] Stephens, C, Arena, A., and Gupta, K.: "CFDBased Aeroservoelastic Predictions with Comparisons to Benchmark Experimental Data," AIAA 99-0766, January 1999.

[10] Djayapertapa, L. and Allen, C. B.: "Aeroservoelastic Computations in Unsteady Transonic Flow," AIAA 2000-4226, August 2000.

[11] Guillot, D. M. and Friedmann, P. P.: "A Fundamental Aeroservoelastic Study Combining Unsteady CFD With Adaptive Control," AIAA 941721-CP, April 1994. 
[12] Friedmann, P. P., Guillot, D. M., and Presente, E.: "Adaptive Control of Aeroelastic Instabilities in Transonic Flow and Its Scaling," AIAA 97-0581-CP, January 1997.

[13] Newsom, J. R.: Designing Active Control Laws in a Computational Aeroelasticity Environment. PhD Dissertation, Virginia Tech, April 2002.

[14] Batina, J. T.; Seidel, D. A.; Bland, S. R.; and Bennett, R. M.: "Unsteady Transonic Flow Calculations for Realistic Aircraft Configurations," Journal of Aircraft, Vol.26, No. 1, January 1989, pp. 21-28.

[15] Batina, J. T.: "Efficient Algorithm for Solution of the Unsteady Transonic Small-Disturbance Equation," Journal of Aircraft, Volume 25, July 1988, pp. 598-605.

[16] Juang, J.: "An Overview of Recent Advances in System Identification,” AIAA-93-1664, April 1993

[17] Phan, M Q.;Horta, L. G., Juang, J., and Longman, R. W.: "Identification of Linear Systems by an Asymptotically Stable Observer," NASA TP 3164, June 1992.

[18] Scott, M; Gilbert, M;, and Demeo, M.: "Active Vibration Damping of the Space Shuttle Remote Manipulator System," Journal of Guidance, Control, \& Dynamics., Volume 16, No. 2, Mar-April 1993, pp. 275-280.

[19] Chen, W. and Valasek, J.: "Observer/Kalman Filter Identification for On-line System Identification of Aircraft," AIAA-99-4173, August 1999.

[20] Juang, J.N. and Pappa, R.S.: “An Eigensystem Realization Algorithm for Modal Parameter Identification and Model Reduction," Journal of Guidance, Control, and Dynamics, Vol. 8, No. 5, 1985, pp. 620-627.

[21] Scott, R. C., Hoadley, S. T., Wieseman, C. D., and Durham, M. H.: "The Benchmark Active Controls Technology Model Aerodynamic Data," AIAA 97-0829-CP, January 1997.
[22] Silva, W. A. and Bennett, R. M.: "Investigation of the Aeroelastic Stability of the AFW Wind-Tunnel Model Using CAP-TSD," NASA TM 104142, September 1991.

[23] Bisplinghoff, R., Ashley, H., and Halfman, R.: Aeroelasticity, Addison Wesley Publishing Company, Reading, Massachusetts, 1954.

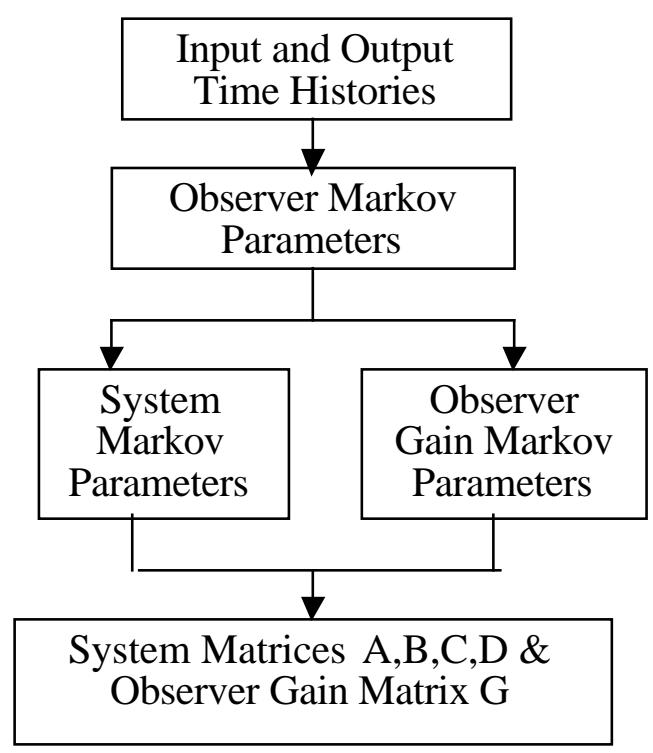

Figure 1. Flow Chart for OKID

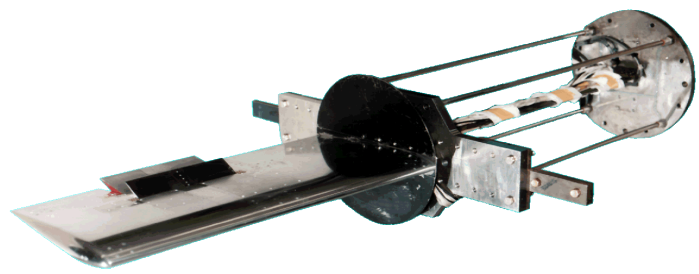

Figure 2. Photograph of BACT Wind-Tunnel Model 


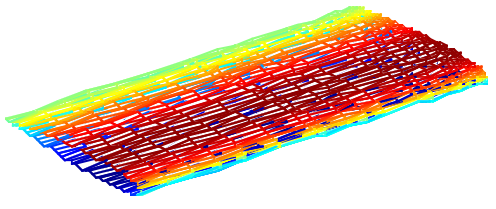

Figure 3. CAP-TSD Mesh of BACT Model
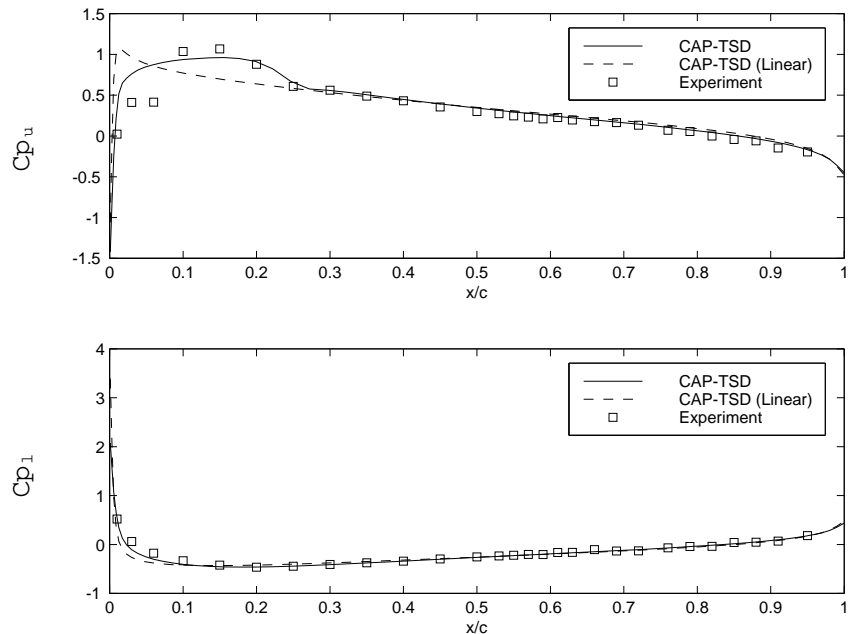

Figure 4. Aerodynamic results $\alpha=2$ degrees, $M=0.77$. $60 \%$ semi-span
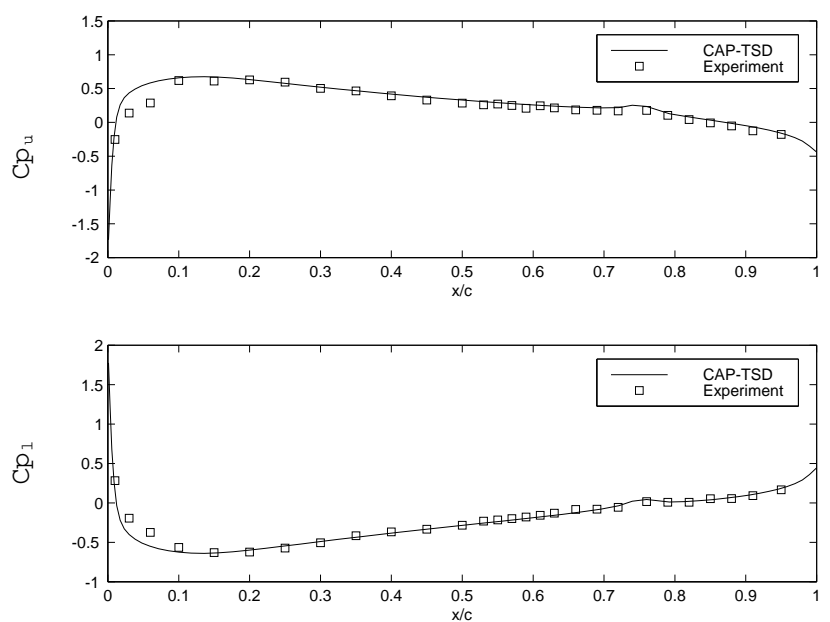

Figure 5. Aerodynamic results $\delta=2$ degrees, $M=0.77$, $60 \%$ semi-span

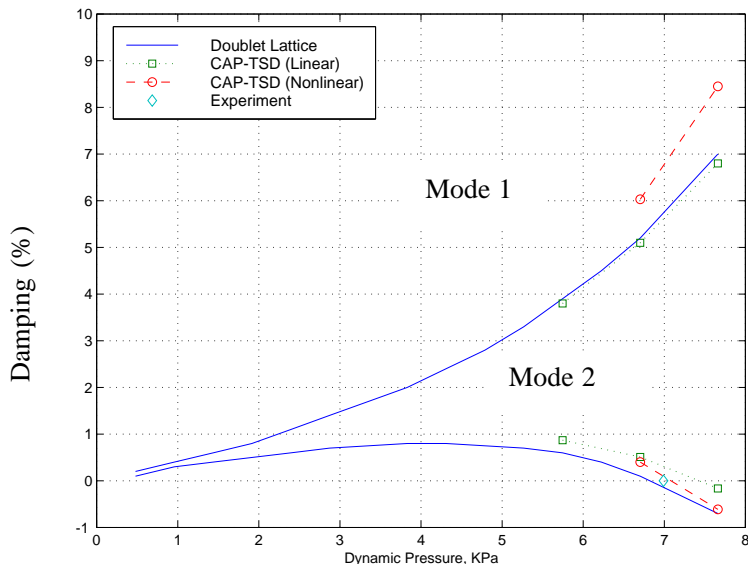

Figure 6. Dampings as a function of dynamic pressure, 3-d

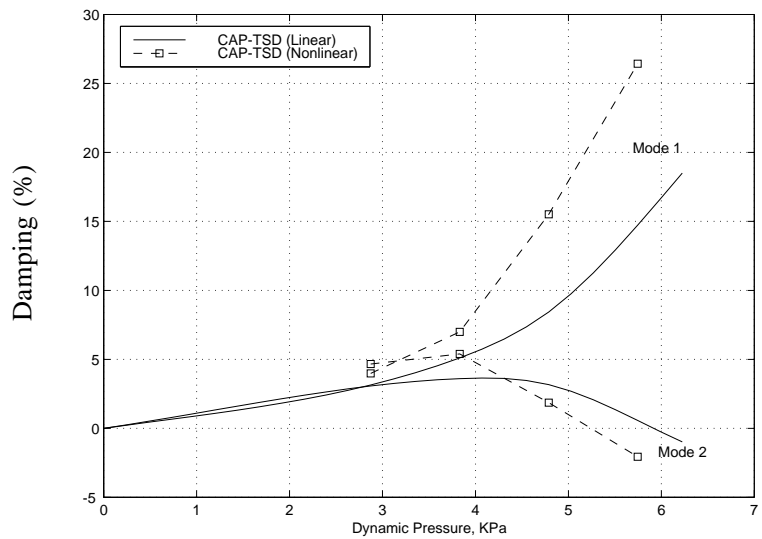

Figure 7. Damping as a function of dynamic pressure, 2-d
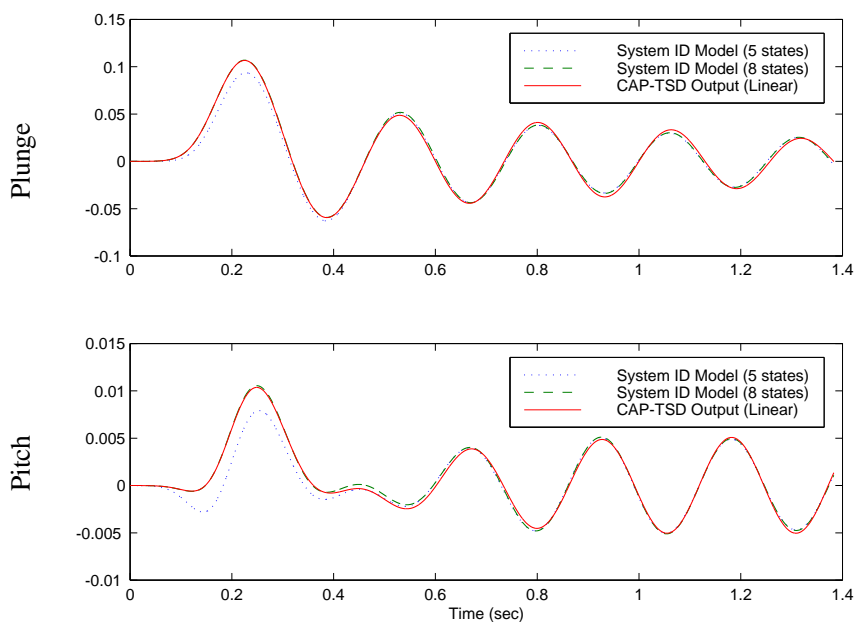

Figure 8. System ID results for displacements, $\alpha=0$ degrees 

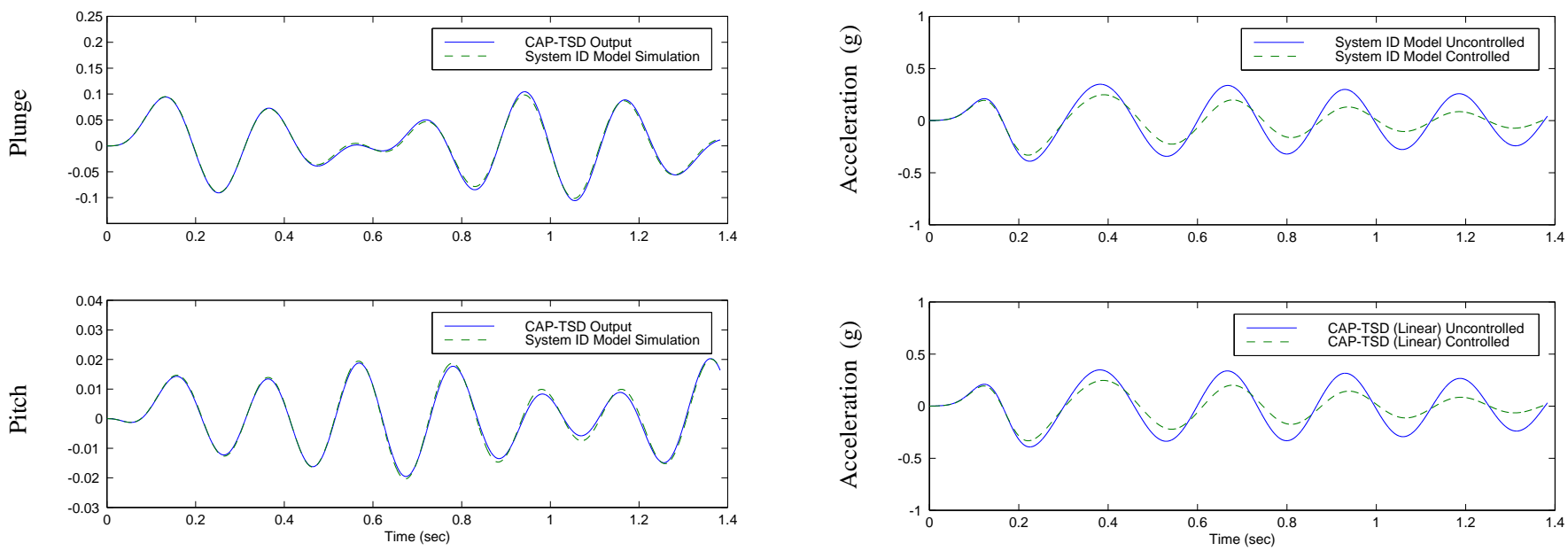

Figure 9 (b). Comparison of displacement responses for a $5 \mathrm{~Hz}$ sine wave input
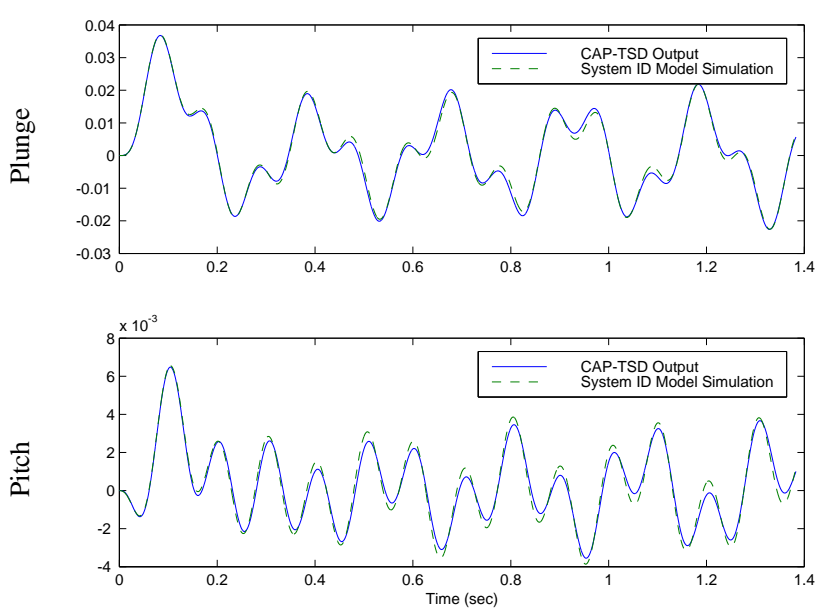

Figure 9 (a). Comparison of displacement responses for a $10 \mathrm{~Hz}$ sine wave input
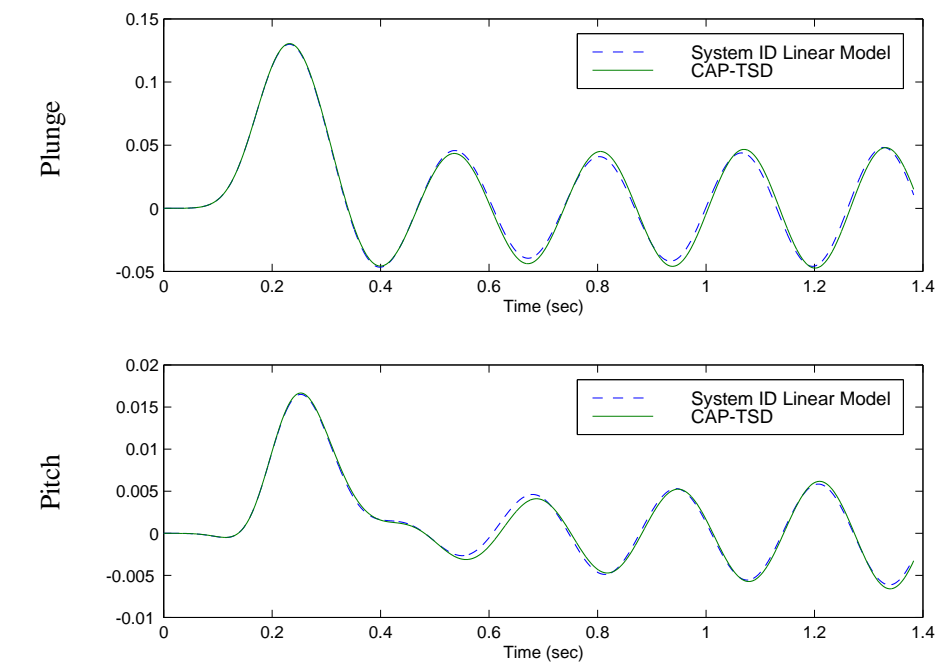

Figure 11. Case 2 system ID results for displacements, $\alpha=0$ degrees 

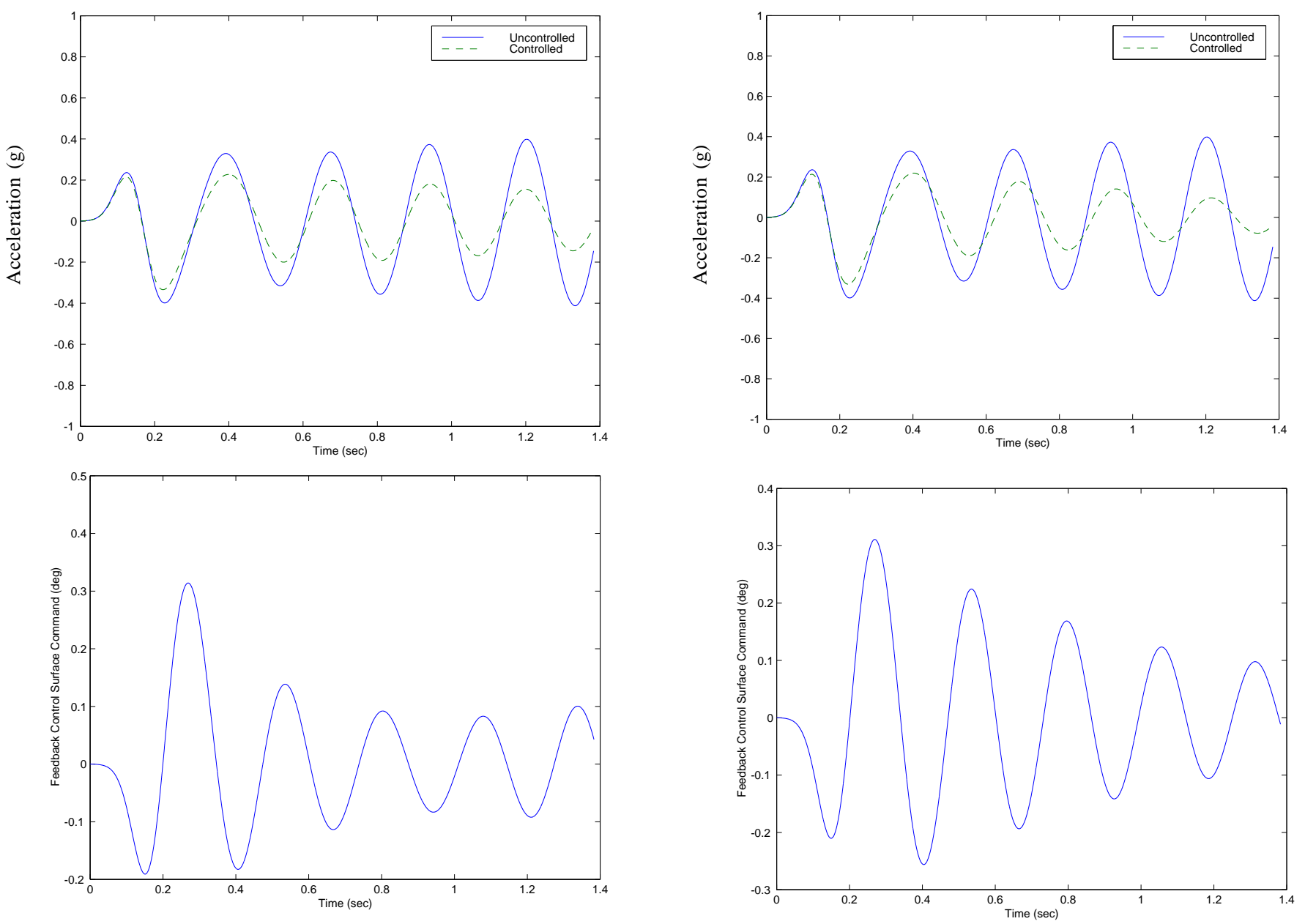

Figure 12. Case 2 controlled results

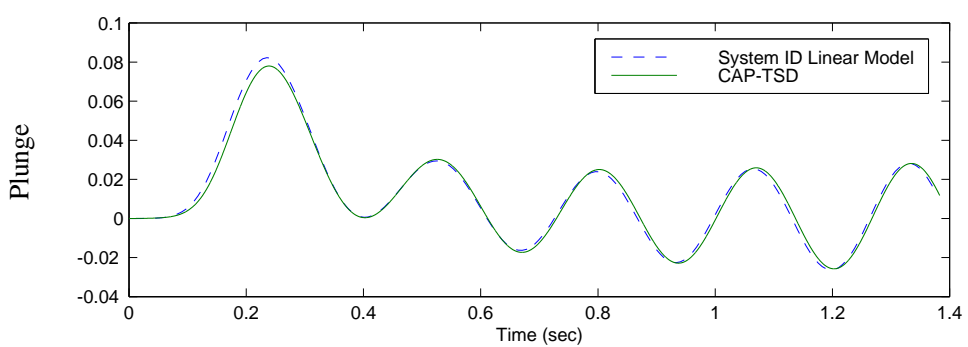

Figure 13. Case 3 controlled results

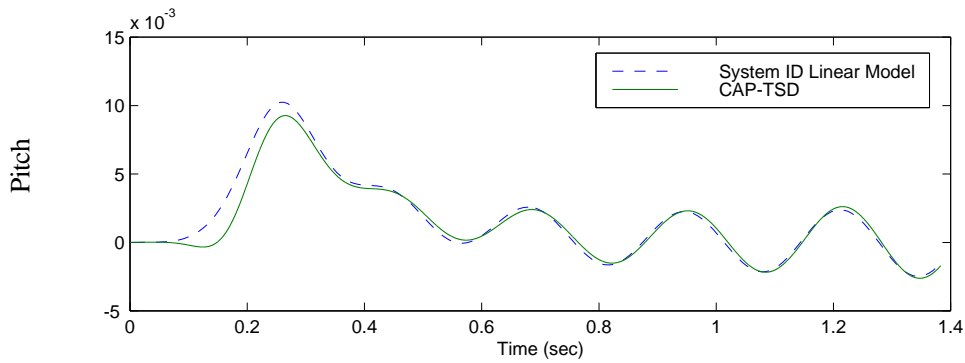

Figure 14. Case 4 system ID results for displacements, $\alpha=.6$ degrees 

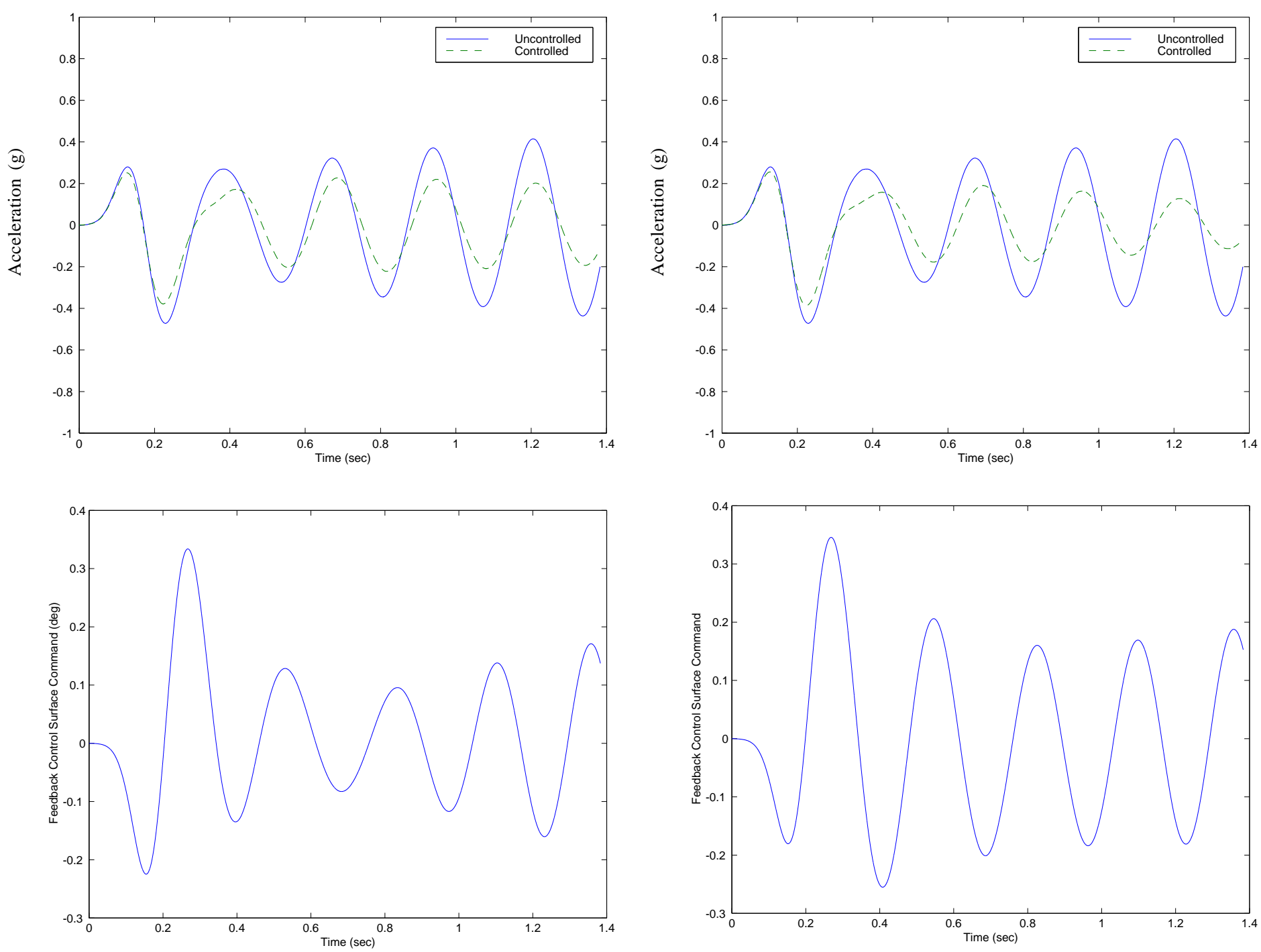

Figure 15. Case 4 controlled results

Figure 16. Case 5 controlled results 